\title{
Effect of Microwave Sterilization on Community Periodontal Index of Treatment Needs Probe Ball Dimension: An Analytical Study
}

\author{
${ }^{1}$ Karibasappa Nagappa, ${ }^{2}$ Vardhaman Jain, ${ }^{3}$ Gaurao Mali, ${ }^{4}$ Digesh Bafiwala, ${ }^{5}$ Jasvender Kour
}

\begin{abstract}
Introduction: The heart of science is measurement. The World Health Organization (WHO) has recommended community periodontal index $(\mathrm{CPI})$ probe to accurately measure the periodontal pocket depth. Repeatedly sterilizing the instrument brings undesirable changes in the material properties, such as alteration in dimensions, and hardness contributing to improper validity. The CPI probe with $0.5 \mathrm{~mm}$ ball end is ideal to measure the exact periodontal pocket depth.
\end{abstract}

Aims and objectives: To assess the changes in the dimension of CPI probe ball end at different cycles of microwave sterilization. To assess and compare the horizontal and vertical dimension of Hu-Friedy PCP11 and GDC PCP11 CPI probes ball end.

Materials and methods: The study was carried at the School of Engineering and Technology, Pune, India. Each community periodontal index of treatment needs (CPITN) probe underwent 100 cycles of microwave sterilization, $1350 \mathrm{~W}$, and $2450 \mathrm{MHz}$ for 16 minutes. After each cycle, the CPI ball dimensions were recorded using manual micrometer and magnifying glass. Unpaired $t$ test was used for statistical analysis.

Results: After 100 cycles of sterilization, circumferential ball dimension of Hu-Friedy measured $0.034 \pm 0.02$ and GDC measured $0.219 \pm 0.028$ and the difference was statistically highly significant $p<0.001$.

Conclusion: Hu-Friedy CPITN probe exhibited better ball dimensional stability and was more valid. Dimensional stability after sterilization procedures is highly relevant for biosafety.

Keywords: Ball end dimension, Community periodontal index probe, Micrometer, Microwave sterilization.

\footnotetext{
${ }^{1}$ Professor, ${ }^{2,3}$ Assistant Professor, ${ }^{4}$ Professor and Dean ${ }^{5}$ Graduate Student (4th Year)

${ }^{1,5}$ Department of Public Health Dentistry, Ajeenkya D Y Patil Dental School, Pune, Maharashtra, India

${ }^{2,3}$ Department of Public Health Dentistry, ACPM Dental College Dhule, Maharashtra, India

${ }^{4}$ Department of Conservative and Dentistry, Ajeenkya D Y Patil Dental School, Pune, Maharashtra, India

Corresponding Author: Karibasappa Nagappa, Professor Department of Public Health Dentistry, Ajeenkya D Y Patil Dental School, Pune, Maharashtra, India, Phone: +912067077710 e-mail: drkb31@gmail.com
}

How to cite this article: Nagappa K, Jain V, Mali G, Bafiwala D, Kour J. Effect of Microwave Sterilization on Community Periodontal Index of Treatment Needs Probe Ball Dimension: An Analytical Study. Int J Experiment Dent Sci 2018;7(1):8-12.

Source of support: Nil

Conflict of interest: None

\section{INTRODUCTION}

The periodontal pocket is the cardinal symptom of periodontitis. As it is rightly said, "The heart of science is measurement." The correct identification and accurate assessment of periodontal pockets are important for the diagnosis of periodontitis. ${ }^{1}$ In addition, quantifying the changes in periodontal pockets is important for the evaluation of disease severity, disease progression, and therapeutic efforts. Periodontal pocket examination should be accurate, technically simple, and quick. To date, the periodontal probe is the only instrument that has been found to be reliable and convenient in pocket examination. ${ }^{2}$ Periodontal probe is universally used to assess the periodontal pocket depth. Multiple periodontal probes are available in market to aid the dental practitioners in diagnosing and planning the periodontal treatment. The joint committee of WHO/Fédération Dentaire Internationale has recommended the CPI probe with ball dimension $0.5 \mathrm{~mm}$ for both clinical practitioners and epidemiologist to plan an appropriate periodontal treatment. ${ }^{3}$

Sterilization of dental instruments helps to prevent the cross-infection. The sterilization efficacy of microwave is as good and identical to the autoclave that is considered to be the gold standard for sterilization. ${ }^{4}$ Hence, in the present study, probes were subjected to microwave sterilization. Many studies have shown, due to repeated cycles of sterilization, the undesirable changes in physical properties of instruments, such as change in dimensional stability, hardness, sharpness, etc., ${ }^{5-7}$ that might result in improper evaluation of the clinical condition. The nature of dental disease, and its regional location, demands the precision of dental instruments in carrying out an effective dental treatment. Any physical change hampers the dentist to perfect the art of dentistry. Studies have been carried out to assess and choose the best method of sterilization on tine diameter of specific probes; ${ }^{3}$ however, 
to the best of our knowledge and after literature search, no studies are carried to assess the effect of sterilization on two different, commonly used CPI probes ball dimension. Thus, the present study was carried with an aim to assess and compare the Hu-Friedy and GDC probe horizontal and vertical ball dimension after repeated cycles of microwave sterilization.

\section{MATERIALS AND METHODS}

The present study was carried out in the Mechanical Department at the School of Engineering and Technology, Ajeenkya D Y Patil University, Pune. Ethical clearance for the study was obtained from the Institutional Ethical Committee.

\section{Step 1}

The material for this study comprised of Hu-Friedy PCP11 and GDC PCP11 unused two types of commonly used periodontal probes. The Senior Professor of Mechanical Department trained the principal investigator $(\mathrm{KB})$ for recording the measurements precisely. After deliberate training and calibration, baseline data of the unused probes ball dimension were recorded using manual micrometer (Mitutoyo Corp., Aurora, Illinois, USA) at the accuracy level of $0.01 \mathrm{~mm}$ and magnifying lens. Eight later periodontal probes were subjected to microwave sterilization.

\section{Step 2}

A domestic microwave oven (LG wonder convection, MC 805AA) was taken and a borosil beaker filled with distilled water containing two different CPI probes was immersed and kept on the turntable of microwave for sterilization. Each microwave cycle was carried out at $1350 \mathrm{~W}, 2450 \mathrm{MHz}$ till 16 minutes, ${ }^{4}$ and after completion of each cycle, ball dimension was measured.

\section{Recording}

While recording measurements, Abbes' principle was adapted. ${ }^{8}$ To overcome any potential changes in measurement due to change in the position, we customized the horizontal and vertical reference point on the ball by placing the colored pointed dot followed by pasting cellophane tape. Throughout the study, the standard reference point marked on the ball was used to record horizontal and vertical ball dimension of ball end. Standardized probe posture was maintained using appropriate adhesive material during the study period by customizing the table end to hold the probe. Care was taken to record measurements with utmost precision throughout after each cycle.
Micrometer frame expands due to heat transfer from hand to frame when the frame is held in the bare hand, which may result in a significant measurement error due to temperature-induced expansion, so gloves were worn. When performing a measurement, to overcome the influence of thermal expansion, note was also taken to allow micrometer standard returns to the original length. ${ }^{8}$

\section{Step 3: How to Read Measurements}

\section{Micrometer with Standard Scale (Graduation: $0.01 \mathrm{~mm}$ )}

The thimble scale can be read directly to $0.01 \mathrm{~mm}$, but may also be estimated to be $0.001 \mathrm{~mm}$ when the lines are nearly coincident because the line thickness is $1 / 5$ of the spacing between them.

Formula to calculate: ${ }^{9}$

Total reading $=$ Main scale reading $+($ circular scale reading $\times \mathrm{LC})$

Least count (LC) for micrometer is $0.01 \mathrm{~mm}$.

\section{Statistical Analysis}

All statistical procedures were performed using Statistical Package for the Social Sciences version 20.0 software (IBM, Armonk, New York, USA). The data exhibited a normal and homogeneous distribution; thus, ball dimensions were analyzed using mean score. Data are presented as mean with $95 \%$ confidence interval. A p-value $<0.05$ was considered statistically significant. The distribution of measurements between two different periodontal probes was compared with the unpaired t-test.

\section{RESULTS}

The results demonstrate that both the probes' ball dimensions were not precise and did not fulfill the ideal requirement of $0.5 \mathrm{~mm}$ as recommended by WHO.

Before the sterilization, Hu-Friedy ball measured horizontally $0.070 \mathrm{~mm}$ and vertically $0.080 \mathrm{~mm}$, while the GDC probe ball measured horizontally $0.260 \mathrm{~mm}$ and vertically $0.270 \mathrm{~mm}$.

However, after 100 cycles of sterilization, circumferential ball dimension of Hu-Friedy measured $0.034 \pm 0.02$ and GDC measured $0.219 \pm 0.028$ and the difference was statistically highly significant, $\mathrm{p}<0.001$. When the ball dimension was assessed at 25th, 50th, 75th, and 100th cycle, there was a linear reduction in the both the probes and the difference was statistically significant, $p<0.001$ (Table 1).

There was no difference between Horizontal and vertical ball dimensions of $\mathrm{Hu}$-Friedy, horizontal measuring $0.031 \pm 0.021$ and vertical $0.037 \pm 0.022$ with $p>0.05$ (Table 2). 
Table 1: Comparison of ball dimensions between two groups

\begin{tabular}{|c|c|c|c|c|c|c|c|}
\hline \multirow{2}{*}{$\begin{array}{l}\text { Sterilization } \\
\text { cycles }\end{array}$} & \multicolumn{2}{|c|}{ Hu-Friedy (I) } & \multicolumn{2}{|c|}{ GDC (II) } & \multicolumn{3}{|c|}{ Grade I vs II } \\
\hline & Mean & $S D$ & Mean & $S D$ & Mean difference & $t$-value & $p$-value \\
\hline $1-25$ & 0.062 & 0.006 & 0.252 & 0.008 & 0.190 & 91.84 & $0.00^{* *}$ \\
\hline $26-50$ & 0.043 & 0.009 & 0.230 & 0.005 & 0.187 & 91.24 & $0.00^{* *}$ \\
\hline $51-75$ & 0.022 & 0.006 & 0.214 & 0.006 & 0.192 & 116.16 & $0.00^{* *}$ \\
\hline $76-100$ & 0.009 & 0.003 & 0.180 & 0.012 & 0.172 & 70.92 & $0.00^{* *}$ \\
\hline Overall & 0.034 & 0.022 & 0.219 & 0.028 & 0.185 & 52.97 & $0.00^{* \star}$ \\
\hline
\end{tabular}

Unpaired $t$ test; ${ }^{* *} p<0.001$, highly significant; SD: Standard deviation

Table 2: Comparison between horizontal and vertical ball dimensions in Hu-Friedy

\begin{tabular}{|c|c|c|c|c|c|c|c|}
\hline \multirow{2}{*}{$\begin{array}{l}\text { Sterilization } \\
\text { cycles }\end{array}$} & \multicolumn{2}{|c|}{ Horizontal } & \multicolumn{2}{|c|}{ Vertical } & \multicolumn{3}{|c|}{ Horizontal vs vertical } \\
\hline & Mean & $S D$ & Mean & $S D$ & Mean difference & $t$-value & $p$-value \\
\hline $1-25$ & 0.059 & 0.008 & 0.066 & 0.004 & 0.006 & 3.46 & $0.002^{*}$ \\
\hline $26-50$ & 0.039 & 0.009 & 0.047 & 0.009 & 0.008 & 3.19 & $0.003^{*}$ \\
\hline $51-75$ & 0.018 & 0.004 & 0.025 & 0.008 & 0.007 & 3.93 & $0.00^{* *}$ \\
\hline $76-100$ & 0.008 & 0.002 & 0.009 & 0.003 & 0.001 & 1.63 & $0.11, \mathrm{NS}$ \\
\hline Overall & 0.031 & 0.021 & 0.037 & 0.022 & 0.006 & 1.83 & $0.07, \mathrm{NS}$ \\
\hline
\end{tabular}

Unpaired t test; ${ }^{*} p<0.05, S ;{ }^{* *} p<0.001$, highly significant; $p>0.05$, NS: Not significant; SD: Standard deviation

Table 3: Comparison between horizontal and vertical ball dimensions in GDC

\begin{tabular}{|c|c|c|c|c|c|c|c|}
\hline \multirow{2}{*}{$\begin{array}{l}\text { Sterilization } \\
\text { cycles }\end{array}$} & \multicolumn{2}{|c|}{ Horizontal } & \multicolumn{2}{|c|}{ Vertical } & \multicolumn{3}{|c|}{ Horizontal vs vertical } \\
\hline & Mean & $S D$ & Mean & $S D$ & Mean difference & $t$-value & $p$-value \\
\hline $1-25$ & 0.248 & 0.008 & 0.257 & 0.009 & 0.009 & 3.64 & $0.001^{*}$ \\
\hline $26-50$ & 0.225 & 0.006 & 0.234 & 0.005 & 0.009 & 5.68 & $0.003^{*}$ \\
\hline $51-75$ & 0.208 & 0.007 & 0.220 & 0.005 & 0.012 & 7.21 & $0.00^{* *}$ \\
\hline $76-100$ & 0.177 & 0.011 & 0.184 & 0.013 & 0.007 & 1.92 & $0.06, \mathrm{NS}$ \\
\hline Overall & 0.215 & 0.027 & 0.224 & 0.028 & 0.009 & 2.29 & $0.02^{*}$ \\
\hline
\end{tabular}

Unpaired t test; ${ }^{*} p<0.05$, significant; ${ }^{* *} p<0.001$, highly significant; $p>0.05$, NS: Not significant; SD: Standard deviation

Table 4: Horizontal ball dimension between two groups

\begin{tabular}{|c|c|c|c|c|c|c|c|}
\hline \multirow{2}{*}{$\begin{array}{l}\text { Sterilization } \\
\text { cycles }\end{array}$} & \multicolumn{2}{|c|}{ Group I (Hor) } & \multicolumn{2}{|c|}{ Group II (Hor) } & \multicolumn{3}{|c|}{ Grade I vs II } \\
\hline & Mean & $S D$ & Mean & $S D$ & Mean difference & $t$-value & $p$-value \\
\hline $1-25$ & 0.059 & 0.008 & 0.248 & 0.008 & 0.189 & 83.26 & $00.00^{* *}$ \\
\hline $26-50$ & 0.039 & 0.009 & 0.225 & 0.006 & 0.186 & 88.67 & $00.00^{* *}$ \\
\hline $51-75$ & 0.018 & 0.004 & 0.208 & 0.007 & 0.190 & 121.3 & $00.00^{* *}$ \\
\hline 76-100 & 0.008 & 0.002 & 0.177 & 0.011 & 0.169 & 75.93 & $00.00^{* *}$ \\
\hline Overall & 0.031 & 0.021 & 0.215 & 0.027 & 0.184 & 53.63 & $00.00^{* *}$ \\
\hline
\end{tabular}

Unpaired $\mathrm{t}$ test; ${ }^{* *} \mathrm{p}<0.001$, highly significant; SD: Standard deviation

Table 5: Vertical ball dimension between two groups

\begin{tabular}{|c|c|c|c|c|c|c|c|}
\hline \multirow{2}{*}{$\begin{array}{l}\text { Sterilization } \\
\text { cycles }\end{array}$} & \multicolumn{2}{|c|}{ Group I (Vert) } & \multicolumn{2}{|c|}{ Group II (Vert) } & \multicolumn{3}{|c|}{ Grade I vs II } \\
\hline & Mean & $S D$ & Mean & $S D$ & Mean difference & $t$-value & $p$-value \\
\hline $1-25$ & 0.066 & 0.004 & 0.257 & 0.009 & 0.191 & 96.25 & $00.00^{* *}$ \\
\hline $26-50$ & 0.047 & 0.009 & 0.234 & 0.005 & 0.187 & 93.04 & $00.00^{* *}$ \\
\hline $51-75$ & 0.025 & 0.008 & 0.220 & 0.005 & 0.195 & 106.36 & $00.00^{* *}$ \\
\hline $76-100$ & 0.009 & 0.003 & 0.184 & 0.013 & 0.175 & 66.34 & $00.00^{* *}$ \\
\hline Overall & 0.037 & 0.022 & 0.224 & 0.028 & 0.187 & 52.16 & $00.00^{* *}$ \\
\hline
\end{tabular}

Unpaired t test; ${ }^{* *} p<0.001$, highly significant; SD: Standard deviation

However, there was a significant difference between horizontal and vertical ball dimensions of GDC with horizontal measuring $0.215 \pm 0.027$ and vertical $0.224 \pm$ 0.028 , and the difference was statistically significant, $\mathrm{p}<0.05$ (Table 3).
When the intergroup horizontal dimension comparison was tested Hu-Friedy measured $0.031 \pm 0.021$ and GDC measured $0.215 \pm 0.027$, while vertical measurement of $\mathrm{Hu}$-Friedy was $0.037 \pm 0.022$ and GDC measured $0.224 \pm 0.028$, and the difference was highly significant $(\mathrm{p}<0.001)$ (Tables 4 and 5$)$. 


\section{DISCUSSION}

Determining the probing pocket depth is considered as an important method to assess the periodontal health status and monitor the prognosis. Many generation periodontal probes are available; however, WHO has recommended the usage of CPITN probe with ball end $0.5 \mathrm{~mm}$. Worldwide, this helps to compare the prevalence of periodontal treatment needs. The design of a periodontal probe with a sphere end aims to facilitate the detection of subgingival calculus and irregularities in restoration edges, besides providing the patient with more comfort. ${ }^{3}$ Due to the sparse scientific literature available, exact and valid comparison of our study findings could not be compared with other findings; however, a sincere and valid attempt is made to discuss the present study findings.

The present study at baseline demonstrates that neither Hu-Friedy nor GDC probe met the ideal ball dimension, as circumferential ball dimension measured 0.070 and $0.260 \mathrm{~mm}$ respectively. This finding is similar to the studies conducted by Rapp et al. ${ }^{3}$ This calls for introspection, as manufacturers before marketing the probe should fulfill the ideal physical requisite of probes. The present findings also alarm the dentist to choose the precise periodontal probe.

When we compare the baseline ball dimension with the findings obtained after 100th cycle of sterilization, it was seen that the GDC probe has significant reduction compared with the Hu-Friedy. After sterilization, there was a significant and linear reduction in the ball dimension of GDC compared with the Hu-Friedy; this contradicts the manufacturers' claim of probe being nonfading and nonflake. The present findings of GDC probe contradict the GDC manufacturer's claim of superiority even though they claim to use martensitic stainless steel (subjected to hardening treatments) for noncutting tips: AISI 420-class 4. This kind of stainless steel grants good resilience, a very good mechanical resistance to traction and torsion, and excellent resistance to wear and corrosion. ${ }^{10}$ Both the manufacturers claim to be superior and excellent in quality of their product; however, the present study findings throw insight and call for quality check and audit by professionals. The exact reason for better dimensional stability of Hu-Friedy should be subjected to further exploration.

As the three-dimensional shape of the tip of the probe is paramount for appropriate diagnosis, ${ }^{3}$ in the present study, we measured the probe ball in both horizontal and vertical dimension. There was a significant reduction in circumferential, horizontal, and vertical ball dimension of both the probes.

The horizontal dimension reduction was more compared with the vertical dimension, and it was highly significant after the 50th cycle of sterilization, $p<0.001$ (Table 2 and 3). Multiple studies ${ }^{11-13}$ showed the discrepancy in the physical properties of dental instruments but contradict our findings as the Hu-Friedy probe showed maximum dimensional stability. The process of fabricating the probes might differ between the two probes with respect to type of raw material used, methodology of fabrication, and finishing the product. Even though standardized force as recommended by Mitutoyo (at less than 100,000 revolutions for measuring within the guaranteed accuracy range) was used throughout the study, ${ }^{8}$ changes seen might be attributed to human error while fine-tuning the closing ends of the micrometer to record the ball end measurements.

When the horizontal and vertical ball dimensions of Hu-Friedy and GDC were compared, more reduction was seen in GDC and the difference was statistically highly significant, $\mathrm{p}<0.001$ (Tables 4 and 5) at 25th to 100 th cycle. Errors in visual assessment, rounding off to the nearest $\mathrm{mm}$, recording errors, effect of water, probe composition and microwave irradiation on probes might be the reasons. According to Miller, ${ }^{14}$ irrespective of the process used to achieve sterilization, corrosion will occur more quickly in an autoclave or in any other environment involving water and heat. Further studies have to be carried out to ascertain the probable reasons in variations of ball dimensions.

In our study, we did not use the testing probes to clinically check the periodontal pocket in between sterilization cycles, as we believed that variation in probing, probing force, subgingival calculus, root configuration, and root cementum might influence the stability of ball dimensions. Microwave sterilization was preferred ${ }^{15}$ over autoclave to overcome the time constraint and manual micrometer was used due to its easy availability; these could have influenced the study findings and thus, we recommend further studies to overcome these inherent limitations of the study.

Shelf life of living or nonliving items cannot be discounted and it comes true through our research findings. The present study throws an important insight regarding the timing of changing the periodontal probe, after 50 cycles of sterilization; periodontal probe usage is questionable due to significant change in the ball dimension. Ignoring the durability and continuous usage of the periodontal probes will result in impairment of recordings, resulting in improper diagnosis of periodontal health status. So, we conclude that the ball diameter accuracy should be considered in addition to other variables in relation to periodontal probing, particularly for clinical research. Standardization of tine characteristics and avoidance of the use of different types or batches in a single study should enhance the accuracy and reproducibility of 
periodontal probe-dependent measurements. Furthermore, the results of this study should be interpreted with caution because the use of microwave is not still recognized by any standards as a sterilization method and may not encourage some clinicians to consider off-label use of these devices in clinical practice. However, microwave sterilization and comparison among dry heat, autoclave, and microwave irradiation should be the subject of further investigations.

\section{CONCLUSION}

The conclusion from this study opens the ways to further evaluate the accuracy of diagnostic aids. Till date, the literature being scant about the observations for larger sample size, there exists a need for studies to evaluate the various diagnostic aids using sophisticated measures to confirm and help manufacturers to increase the accuracy and thus the reliability of gold standard diagnostic aids. The present research opens new vista to assess the exact timing to discontinue the use of periodontal probes.

\section{REFERENCES}

1. Hefti AF. Periodontal probing. Crit Rev Oral Biol Med 1997 Jul;8(3):336-356.

2. Khan S, Cabanilla LL. Periodontal probing depth measurement: a review. Compend Contin Educ Dent 2009 Jan-Feb; 30(1):12-14.

3. Rapp GE, Barbosa Júnior Ade A, Mendes AJ,Motta AC, BiãoMA, Garcia RV. Technical assessment of WHO-621 periodontal probe made in Brazil. Braz Dent J 2002 Feb;13(1):61-65

4. Karibasappa GN, Sujatha A, Singh R, Prithiani P, Rajeshwari K. Microwave-a novel wave in dentistry. IOSR J Dent Med Sci (IOSR-JDMS) 2013 Nov-Dec;12(3):1-5.
5. PortoAN,BorgesÁH,Semenoff-SegundoA,RaslanSA,PedroFL, Jorge AO, Bandeca MC. Effect of repeated sterilization cycles on the physical properties of scaling instruments: a scanning electron microscopy study. J Int Oral Health 2015 May;7(5):1-4.

6. Bapna MS, Mueller HJ. Corrosion of dental burs in sterilizing and disinfecting solutions. J Prosthet Dent 1988 Apr;59(4):503-511.

7. Cooley RL, Marshall TD, Young JM, Huddleston AM. Effect of sterilization on the strength and cutting efficiency of twist drills. Quintessence Int 1990 Nov;21(11):919-923.

8. Micrometers. Quick guide to precision measuring instruments. [cited 2017 Feb 5]. Available from: www.mitutoyo. co.jp/eng/products/menu/QuickGuide_Micrometers.pdf.

9. Gupta, IC. Linear measurements. In: A text book of engineering metrology. 7th ed. New Delhi: Dhanpat Rai Publications (p) Ltd; 2010. pp. 140-169.

10. GDC-Marketing. Diagnostic probes-GDC marketing importers and exporters of dental. [cited 2017 Feb 5]. Available from: www.gdc-marketing.com/product.php?Sid=116\&cid $=44$.

11. Ashok KT, Mahesh MN, Kshitij Vipin P, Arunkumar M, Arvind AA, Suresh MH. Reliability and accuracy of gold standard diagnostic aid for periodontal disease: an observational study. Int J Dent Health Sci 2016 Apr;3(2):327-335.

12. Batista Mendes GC, Padovan LE, Ribeiro-Júnior PD, Sartori EM, Valgas L, Claudino M. Influence of implant drill materials on wear, deformation, and roughness after repeated drilling and sterilization. Implant Dent 2014 Apr;23(2):188-194.

13. Geaman JR, Moser JB. Hardness and metallurgical characterization of dental hand-cutting instruments. Dent Mater 1987 Oct;3(5):252-255.

14. Miller $\mathrm{CH}$. Tips on preparing instruments for sterilization. Am J Dent 2002 Feb;15(1):66.

15. Alexandrou G, Chrissafis K, Vasiliadis L, Pavlidou E, Polychroniadis EK. Effect of heat sterilization on surface characteristics and microstructure of Mani NRT rotary nickel-titanium instruments. Int Endod J 2006 Oct;39(10): 770-778. 